\title{
On the Design of Robust Deadbeat Regulators
}

\author{
James Lam Hom Kin Tso \\ Department of Mechanical Engineering \\ University of Hong Kong \\ Pokfulam Road, HONG KONG
}

\author{
Nam Kiu Tsing \\ Department of Mathematics \\ University of Hong Kong \\ Pokfulam Road, HONG KONG
}

\begin{abstract}
This paper considers the synthesis of state feedback gains which provide robustness against perturbation in deadbeat regulation. It is formulated as an unconstrained optimization problem. Through a posteriori perturbation analysis of the closed-loop eigenvalues, the justification of the use of a new objective function to measure the robustness of deadbeat systems is established. The objective function does not require the computation of eigenvectors and has simple analytical gradient and Hessian. A numerical example is employed to illustrate the effectiveness of the proposed method.
\end{abstract}

\section{Introduction}

For a completely state controllable system, it is well known that the closed-loop poles via state-feedback can be assigned at any set of self-conjugate complex numbers (Petkov, Christov, and Konstantinov 1991). The statefeedback gain matrix, except in the single-input case, is in general nonunique for a given set of desired closedloop poles. A classical application of this pole assignment technique in the discrete-time is to have the closedloop poles all positioned at the origin. In this case, the system exhibits a deadbeat characteristics such that the zero-input response of the system dies down to zero in a finite time steps. This deadbeat control problem was considered by Kalman and Bertram (1958) for the singleinput case. The multivariable case was later addressed by many, Farison and Fu (1970), Kučera (1971), Mullis (1972), Leden (1977), Emami-Naeini and Franklin (1982), to name a few. More recently, a recursive algorithm was proposed by van Dooren which allows the computation of minimum norm deadbeat regulator gains efficiently (van Dooren 1984). Different classes of deadbeat controllers were considered by Fahmy and O'Reilly (1983a) using the eigenstructure assignment approach. This approach was exploited to designing minimum norm deadbeat systems (Fahmy and O'Reilly 1983b). The minimal parametrization of a class of state-feedback deadbeat controllers was given by Schlegel (1982). The nonuniqueness of the statefeedback gain matrix allows one to search for a solution which leads to, in certain sense, a more robust closedloop system. This is especially important to deadbeat control where the closed-loop state matrix is nilpotent and in general has a nontrivial Jordan structure, hence more susceptible to large errors when perturbed (Stewart and Sun 1990). Many methods have been proposed on the choice of the state-feedback gain matrix for pole assignment. A large number of methods for robust eigen0-7803-3590-2/96 \$5.00 @ 1996 IEEE value/eigenstructure assignment are formulated as minimization problems with objective functions measuring either the conditioning of the eigenvalue/eigenstructure of closed-loop state matrix (Cavin III and Bhattacharyya 1983; Byers and Nash 1989; Kautsky and Nichols 1985; Kautsky, Nichols, and van Dooren 1985; Lam and Yan 1995) or eigenvalue differential sensitivity (Gourishankar and Ramar 1976; Owens and O'Reilly 1989) (a comprehensive list of methods and references can be found in (White 1995)). Unfortunately, the robust deadbeat pole assignment problem has not been directly addressed. In particular, some of the measures of robustness may not even be valid for repeated eigenvalues with nontrivial Jordan structures.

In this paper, we tackle the robust deadbeat regulation problem via the unconstrained minimization of a new objective function measuring the spectral variation of the closed-loop system. The objective function is specific to deadbeat closed-loop systems. The gradient and the Hessian matrix of the objective function are derived for use in standard minimization routines.

This paper is divided into five sections. Basic properties of the deadbeat regulation problem and preliminaries results are given in Section 2. A posteriori pertrubation analysis on the closed-loop eigenvalues is given to justify the use of a new objective function to measure the robustness in deadbeat systems. The robust deadbeat regulation problem is formulated in Section 3 as an unconstrained minimization problem. The number of minimization variables are discussed. Analytical formulas for minimization are also derived and the procedures are summarized in the form of an algorithm. In Section 4, we use a numerical example to demonstrate the effectiveness of the computed optimal feedback gain. Finally, concluding remarks are given in Section 5 .

\section{Preliminary Results}

Consider a discrete-time finite-dimensional linear timeinvariant system with $q(q>1)$ inputs described by

$$
x_{t+1}=A x_{t}+B u_{t} \quad t=0,1,2, \ldots
$$

where $A \in \mathbb{R}^{n \times n}, B \in \mathbb{R}^{n \times q}, x_{t} \in \mathbb{R}^{n \times 1}, u_{t} \in \mathbb{R}^{q \times 1}$. The input matrix $B$ is assumed to have full column rank and the pair $(A, B)$ is assumed to be reachable which is equivalent to the condition

$$
\operatorname{rank}\left(\left[\begin{array}{cccc}
B & A B & \cdots & A^{n-1} B
\end{array}\right]\right)=n
$$


By applying a time-invariant state-feedback law

$$
u_{t}=K x_{t}+v_{t}
$$

where $v_{t}$ is the new reference input, there results the closed-loop system given by

$$
x_{t+1}=(A+B K) x_{t}+B v_{t}
$$

Under the reachability assumption of $(A, B)$, the spectrum of the closed-loop state matrix $A+B K$ can be assigned to any arbitrary set of self-conjugate complex number of cardinality $n$ by proper choice of the feedback gain matrix $K \in \mathbb{R}^{q \times n}$. With $\operatorname{rank}(B)>1$, the choice of $K$ is nonunique. To exhibit deadbeat zero-input response, the closed-loop state matrix is designed to be a nilpotent matrix, that is, $(A+B K)^{m}=0$ for some positive integer $m$. For fast regulation, $K$ should be chosen such that $m$ is the smallest possible. To simplify matters, we assume that $A$ has no zero eigenvalues. The Robust Deadbeat Regulation ( $R D R$ ) problem is then to find $K$ such that the closed-loop poles are all assigned at the origin of the complex plane with $m$ as small as possible, the closed-loop system is made to be as robust as possible under certain measures.

In the following, we will consider the relationship between $m$, the Jordan structure of $A+B K$, and the measure of robustness in face of perturbations or uncertainties in the closed-loop state matrix.

\subsection{Minimum Time Regulation}

Let

$$
B=\left[\begin{array}{llll}
b_{1} & b_{2} & \cdots & b_{q}
\end{array}\right]
$$

Consider the reachability matrix $P$ given by

$$
\begin{aligned}
& P=\left[\begin{array}{llll}
B & A B & \cdots & A^{n-1} B
\end{array}\right] \\
& =\left[\begin{array}{lllllll}
b_{1} & \cdots & b_{q} & A b_{1} & \cdots & A b_{q} & \cdots
\end{array}\right. \\
& \left.A^{n-1} b_{1} \quad \cdots \quad A^{n-1} b_{m}\right]
\end{aligned}
$$

This matrix has rank $n$. Suppose the linearly independent columns of $P$ are chosen in order from left to right and are rearranged as

$$
\begin{array}{lllllll}
b_{1} & A b_{1} & \cdots & A^{k_{1}-1} b_{1} & b_{2} & A b_{2} & \cdots \\
& A^{k_{2}-1} b_{2} & \cdots & b_{q} & A b_{q} & \cdots & A^{k_{3}-1} b_{q}
\end{array}
$$

The nonnegative integers $k_{1}, k_{2}, \ldots, k_{q}$ are known as the Kronecker invariants of the pair $(A, B)$ and more commonly as the reachability indices when arranged in descending order of magnitude (Petkor. Christov and Konstantinov 1991). In general, one has $\sum_{i=1}^{q} k_{i} \leq n$ with equality holds if and only if $(A, B)$ is a reachable pair. The value

$$
k:=\max \left\{k_{1}, i=1,2, \ldots q\right\}
$$

is called the reachability inder of the system. Cotice that if any $k_{i}=0$. then the corresponding chain of vectors in (1) will be absent. but it is still included in the enumeration.

We state the following lemma which can be obtained by the construction as given in Kaczorek (1992. Ihm.3.4) or Goodwin and Sin 1984. Chap. 2.
Lemma 1 Under the assumptions and notation in this section, the state matrix $A+B K$ can be made similar to the nilpotent matrix

$$
J=\operatorname{diag}\left(J_{1}, J_{2}, \ldots, J_{q}\right)
$$

where

$$
J_{i}=\left[\begin{array}{ccccc}
0 & 1 & 0 & \ldots & 0 \\
\vdots & \ddots & \ddots & \ddots & \vdots \\
\vdots & & \ddots & \ddots & 0 \\
\vdots & & & \ddots & 1 \\
0 & \ldots & \ldots & \ldots & 0
\end{array}\right]
$$

is a Jordan block of dimension $k_{i}$ (for $k_{i}=0, J_{i}$ does not appear in $J$ ). Furthermore, apart from a re-ordering of the Jordan blocks, the size of each Jordan block in $J$ is minimal.

Remark 1 Suppose $K$ is chosen such that $A+B K$ is nilpotent. Then the smallest possible size of the largest Jordan block in $A+B K$ has dimension equal to the largest values among the reachability indices which is the reachability index $k$ of the pair $(A, B)$. In other words, the smallest choice of $m$ is equal to $k$ which is the smallest positive integer such that $(A+B K)^{k}=0$. Notice that it is possible to construct a $K$ such that the nilpotent state matrix $A+B K$ has a Jordan block size larger than $k$. However, this is not preferred due to (1) the sensitivity of the zero eigenvalues, and (2) the order of nilpotence equals the number of sampling periods for the closed-loop system to attain the zero state. Furthermore, the shortest regulation time for every initial state corresponds to a Jordan structure with block sizes equal to the reachability indices of the system (Kuč era and S ebek 1984).

Remark 2 It is worth noting that if $k=1$, then there exists a $K$ such that $A+B K=0$. However, this is only possible if $q=n$ (full column rank of $B$ is assumed) and $K=-B^{-1} A$ is the unique solution.

For reason explained in Remark 2, from here onwards we assume $k>1$.

\subsection{Measures of Robustness}

A number of a posteriori measures of robustness will be introduced in this section. These measures are used as objective functions for minimization as well as for comparing different solutions. A common measure of rotustness for a matrix in face of perturbations is the condition number of its eigenvector matrix. The following theorem gives a quantitative description of the variation of eigenvalues for a matrix 11 with perturbation $\Delta$ which can be found in Chatelin (1993. Thm.4.4.2) and Stewart and Sun (1390. Chap.4.Thm.1.12). 
Theorem 1 For each eigenvalue $\lambda$ of $M+\Delta$, there exists an eigenvalue $\mu$ of $M$, such that

1. If $M$ is nondefective, then

$$
|\lambda-\mu| \leq\|T\|_{2}\left\|T^{-1}\right\|_{2}\|\Delta\|_{2}
$$

where $T$ is an eigenvector matrix of $M$

2. If $M$ is defective, then

$$
\frac{|\lambda-\mu|^{m}}{1+|\lambda-\mu|+\cdots+|\lambda-\mu|^{m-1}} \leq\|T\|_{2}\left\|T^{-1}\right\|_{2}\|\Delta\|_{2}
$$

where $T$ is a generalized eigenvector matrix of $M$ (that is, $T^{-1} M T=J$ where $J$ is the Jordan canonical form of $M$ ) and $m$ is the size of the largest Jordan block of $M$.

The result in (5) is generally known as the Bauer-Fike theorem. In the case where $M=A+B K$ is nilpotent, we have $\mu=0$. Consequently, (5) and (6) reduce to respectively .

$$
|\lambda| \leq\|T\|_{2}\left\|T^{-1}\right\|_{2}\|\Delta\|_{2}
$$

and

$$
\frac{|\lambda|^{m}}{1+|\lambda|+\cdots+|\lambda|^{m-1}} \leq\|T\|_{2}\left\|T^{-1}\right\|_{2}\|\Delta\|_{2}
$$

These results justify the fact that $\|T\|_{2}\left\|T^{-1}\right\|_{2}$ is often taken as a measure of the conditioning of the spectrum of a matrix. However, the spectral condition number $\kappa_{2}(T):=$ $\|T\|_{2}\left\|T^{-1}\right\|_{2}$ is a nondifferentiable function with respect to $T$. This presents problems in optimization. Therefore, very often the Frobenius condition number

$$
\kappa_{F}(T):=\|T\|_{F}\left\|T^{-1}\right\|_{F}=\sqrt{\operatorname{tr}\left(T^{*} T\right) \operatorname{tr}\left(T^{-*} T^{-1}\right)}
$$

is considered since $\kappa_{F}(T)$ is differentiable with respect to $T$. Moreover, we have $\kappa_{2}(T) \leq \kappa_{F}(T)$.

For the robust deadbeat regulator problem, we propose a new measure of robustness specific to this type of problems. The following theorem describes of the variation of the eigenvalues in terms of the spectral norm and the size of the largest Jordan block in a nilpotent matrix $M$.

Theorem 2 Let $M \in \mathbb{R}^{n \times n}$ be nilpotent and $k \geq 1$ be the smallest integer such that $M^{k}=0$. Suppose $\lambda$ is a nonzero eigenvalue of $M+\Delta$ where $\Delta \in \mathbb{R}^{n \times n}$, then

(i) $|\lambda| \leq \max _{i=0, \ldots, k-1}\left\{\left(k\left\|M^{i} \Delta\right\|_{2}\right)^{\frac{1}{i+1}}\right\}$,

(ii) $|\lambda| \leq \max _{i=, \ldots, k-1}\left\{\left(k\left\|M^{i}\right\|_{2}\|\Delta\|_{2}\right)^{\frac{1}{i+1}}\right\}$,

(iii) $|\lambda| \leq \max _{i=0, \ldots, k-1}\left\{\left(k\|M\|_{2}^{i}\|\Delta\|_{2}\right)^{\frac{1}{i+1}}\right\}$.

Proof: Let $v$ be an eigenvector corresponding to the nonzero eigenvalue $\lambda$ of $M+\Delta$. Then

$$
\begin{aligned}
v & =(\lambda I-M)^{-1} \Delta v \\
& =\left(\frac{1}{\lambda} \Delta+\frac{1}{\lambda^{2}} M \Delta+\cdots+\frac{1}{\lambda^{k}} M^{k-1} \Delta\right) v
\end{aligned}
$$

which gives

$$
\begin{aligned}
\lambda^{k} v & =\left(\lambda^{k-1} \Delta+\lambda^{k-2} M \Delta+\cdots+M^{k-1} \Delta\right) v \\
\Longrightarrow|\lambda|^{k} & \leq \sum_{i=0}^{k-1}|\lambda|^{k-1-i}\left\|M^{i} \Delta\right\|_{2}
\end{aligned}
$$

Hence at least one of the following inequalities must hold:

$$
|\lambda|^{k} \leq k|\lambda|^{k-1-i}\left\|M^{i} \Delta\right\|_{2} \quad(i=0, \ldots, k-1)
$$

These inequalities are equivalent respectively to $|\lambda|^{i+1} \leq$ $k\left\|M^{i} \Delta\right\|_{2}(i=0, \ldots, k-1)$, which are in turn equivalent respectively to $|\lambda| \leq\left(k\left\|M^{i} \Delta\right\|_{2}\right)^{\frac{1}{i+1}}(i=0, \ldots, k-1)$. Therefore (i) holds. The inequalities (ii) and (iii) follow from (i).

In the above, the value $k$ is the same as the size of largest Jordan block in the Jordan form of $M$. This fact is used to establish the following proposition.

Proposition 1 With the notation in Theorem 2, let $M=$ $T J T^{-1}$ be a Jordan canonical decomposition where $T$ is a generalized eigenvector matrix of $M$, and

$$
J=\operatorname{diag}\left(J_{1}, J_{2}, \ldots, J_{r}\right)
$$

where

$$
J_{i}=\left[\begin{array}{ccccc}
0 & 1 & 0 & \ldots & 0 \\
\vdots & \ddots & \ddots & \ddots & \vdots \\
\vdots & & \ddots & \ddots & 0 \\
\vdots & & & \ddots & 1 \\
0 & \ldots & \ldots & \ldots & 0
\end{array}\right]
$$

is a Jordan block of dimension $m_{i}$ such that $m_{1}+m_{2}+$ $\cdots+m_{r}=n$ and $k=\max _{i=1}^{r} m_{i}$. Then

$$
\begin{aligned}
& |\lambda| \leq(1+\epsilon)\left\|M^{k-1}\right\|_{2}^{\frac{1}{k}}\|\Delta\|_{2}^{\frac{1}{k}} \leq(1+\epsilon) \kappa_{2}(T)^{\frac{1}{k}}\|\Delta\|_{2}^{\frac{1}{k}} \\
& \text { where } \epsilon=\mathcal{O}(|\lambda|) \rightarrow 0 \text { as }\|\Delta\|_{2} \rightarrow 0 .
\end{aligned}
$$

Proof: Omitted.

Remark 3 It is easy to establish the following inequalities.

$$
\begin{array}{r}
\left\|M^{k-1}\right\|_{2} \leq\left\|M^{k-1}\right\|_{F} \leq \sqrt{\bar{k}} \kappa_{F}(T) \\
\|M\|_{F} \leq \sqrt{n-r} \kappa_{F}(T) \\
\left\|M^{k-1}\right\|_{2} \leq \kappa_{2}(T) \leq \kappa_{F}(T) \\
\left\|M^{k-1}\right\|_{2} \leq\|M\|_{2}^{k-1} \\
\left\|M^{k-1}\right\|_{F} \leq\|M\|_{F}^{k-1}
\end{array}
$$

where $\bar{k}$ is the number of $k \times k$ Jordan blocks $J_{i}, i=$ $1, \ldots, r$ in $J$. These inequalities, together with Theorem 2 and Proposition 1, indicate that $\|M\|_{F}$ may be used as a measure of robustness against perturbation.

In view on Remark 3, the following objective function is considered:

$$
\phi:=|| M \|_{F}^{2}
$$

The reason for considering $\phi$ is threefold. It is clear that if $\phi$ is small, the upper bound on the perturbation is also small. Moreover, as we shall see in Section 3, there exist formulas for the gradient and the Hessian which can be evaluated efficiently. 


\section{Problem Formulation}

As explained in Remark 1, the choice of $K$ should be such that the size of the Jordan blocks in the Jordan canonical decomposition of $A+B K$ be made equal to the reachability indices. That is, there exists a similarity transformation $T \in \mathbb{R}^{n \times n}$ such that

$$
A+B K=T J T^{-1}
$$

where

$$
J=\operatorname{diag}\left(J_{1}, J_{2}, \ldots, J_{q}\right)
$$

Notice that $J_{i} \in \mathbb{R}^{k_{i} \times k_{i}}$ is defined in (10) and those blocks with $k_{i}=0$ does not appear in $J$. Any re-ordering of $J_{i}$ does not affect the values of $\kappa_{2}(T)$ and $\kappa_{F}(T)$. It is important to realize that for a given $A+B K$, the transformation $T$ is not unique. Observe that (17) can be rewritten as

$$
\begin{aligned}
A T-T J+B G & =0 \\
G & =K T
\end{aligned}
$$

Since the eigenvalues of $A$ (open-loop poles) and those of $J$ (closed-loop poles) are assumed to be different, the Sylvester equation (19) gives a unique $T$ as the solution. It is also known from Bhattacharyya and de Souza (1982) that the set of all $G \in \mathbb{R}^{q \times n}$ for which the solution $T$ to the Sylvester equation (19) be nonsingular is open and dense. (We denote this set by $\mathcal{G}$.) In other words, for almost any choice of $G, T=T(G)$ obtained from (19) is unique and invertible. Thus, $G$ is taken as an optimization parameter to minimize the objective function $\phi$. Once an optimal (or near-optimal) solution $G_{\text {opt }}$ is obtained, the corresponding state feedback gain can be recovered as $K_{\text {opt }}:=G_{\text {opt }} T\left(G_{\text {opt }}\right)^{-1}$. Similar idea is also exploited in the continuous-time robust pole/eigenstructure assignment problem (Cavin III and Bhattacharyya 1983; Lam and Yan 1995).

Formally, the following minimization problem is considered.

$$
\min _{G \in \mathcal{G}}\|M\|_{F}^{2}=\min _{G \in \mathcal{G}}\left\|A+B G T(G)^{-1}\right\|_{F}^{2}
$$

\subsection{Gradient and Hessian Formulas}

In this subsection, we derive the gradient and Hessian formulas for $\phi$ with respect to the parameters in $G$. Let $G=\left[g_{i j}\right]_{q \times n}$ and we have $\frac{\partial G}{\partial g_{i j}}=E_{i j}$ where $E_{i j} \in \mathbb{R}^{q \times n}$ is a matrix with zero elements except a value of one at the $(i, j)$ position. Due to the differentiable nature of the objective function $\phi:=\|M\|_{F}^{2}=\operatorname{tr}\left(M^{T} M\right)$, we have the gradient given by

$$
\nabla_{G} \phi:=\left[\frac{\partial \phi}{\partial g_{i j}}\right]_{q \times n}=2\left[\operatorname{tr}\left(M^{T} \frac{\partial M}{\partial g_{i j}}\right)\right]_{q \times n}
$$

where

$$
\frac{\partial M}{\partial g_{i j}}=B\left(E_{i j}-G T^{-1} \frac{\partial T}{\partial g_{i j}}\right) T^{-1}
$$

and

$$
A \frac{\partial T}{\partial g_{i j}}-\frac{\partial T}{\partial g_{i j}} J+B E_{i j}=0
$$

is obtained by differentiating (19). The Sylvester equation (23) has unique solution since it is assumed that the spectra of $A$ and $J$ are disjoint. It is also important to notice from (23) that $\frac{\partial T}{\partial g_{i j}}$ for $i=1, \ldots, q$ and $j=1, \ldots, n$ are independent of $G$ and hence their values are only required to compute once. The Hessian is given by

$$
\nabla_{G}^{2} \phi:=\left[\frac{\partial^{2} \phi_{1}}{\partial g_{i j} \partial g_{i^{\prime} j^{\prime}}}\right]_{q n \times q n}
$$

with

$$
\frac{\partial^{2} \phi}{\partial g_{i j} \partial g_{i^{\prime} j^{\prime}}}=2 \operatorname{tr}\left(\left(\frac{\partial M}{\partial g_{i j}}\right)^{T} \frac{\partial M}{\partial g_{i^{\prime} j^{\prime}}}+M^{T} \frac{\partial^{2} M}{\partial g_{i j} \partial g_{i^{\prime} j^{\prime}}}\right)
$$

where $\frac{\partial^{2} M}{\partial g_{i j} \partial g_{i^{\prime} j^{\prime}}}$ is given by

$$
\begin{gathered}
B G T^{-1}\left(\frac{\partial T}{\partial g_{i j}} T^{-1} \frac{\partial T}{\partial g_{i^{\prime} j^{\prime}}}+\frac{\partial T}{\partial g_{i^{\prime} j^{\prime}}} T^{-1} \frac{\partial T}{\partial g_{i j}}\right) T^{-1} \\
-B\left(E_{i j} T^{-1} \frac{\partial T}{\partial g_{i^{\prime} j^{\prime}}}+E_{i^{\prime} j^{\prime}} T^{-1} \frac{\partial T}{\partial g_{i j}}\right) T^{-1}
\end{gathered}
$$

\subsection{Number of Effective Parameters in $G$}

It should be pointed out that the parametrization of $K$ in terms of $G$ using (19) and (20) is equivalent to that given in (Fahmy and O'Reilly 1983b). The present way to parametrize gives a more reliable way to compute $K$ and $T$, though less explicit than that given by Fahmy and O'Reilly. This is because it does not require the computation of $A^{-1}$ and its powers.

\section{Suppose}

$$
G=\left[\begin{array}{llll}
G_{1} & G_{2} & \cdots & G_{q}
\end{array}\right] \quad, \quad G_{i} \in \mathbb{R}^{q \times k_{i}}
$$

and let $\underline{k}:=\min _{i} k_{i}$. It was shown in (Fahmy and O'Reilly $1983 \mathrm{~b})$ that $K$ is independent of the last $\underline{k}$ columns in each submatrix $G_{i}$ of the parameter matrix $G$. As a consequence, $G$ contains $n-q \underline{k}$ effective parameter columns in $G$ and $q \underline{k}$ ineffective ones. The values of the elements in these redundant columns can be fixed arbitrarily as long as $T(G)$ defined in (19) is nonsingular. The total number of effective elements in $G$ is thus equal to $q(n-q \underline{k})$. For the objective function $\|M\|_{F}^{2}=\|A+B K\|_{F}^{2}$, the partial derivatives of the gradient (21) are therefore zero with respect to the ineffective elements in $G$. This leads to a substantial reduction in the computation of the gradient vector and the Hessian matrix. Hence, the number of minimization variables is not as large as it might appear. However, it must be realized that the number $q(n-q \underline{k})$ of parameters is not the minimum number (Schlegel 1982; Fahmy and O'Reilly 1983b). 


\subsection{Computation Algorithm for Robust Dead- beat Regulators}

We now suggest the following schematic algorithm for computing robust deadbeat regulator gains.

RDR Algorithm: Given a reachable pair $(A, B)$ with $A$ nonsingular, $J$ of the form (18)and $G(0) \in \mathcal{G}$.

1. Compute and store $\frac{\partial T}{\partial g_{i j}}$ via the Sylvester equation (23) for all effective parameter elements $g_{i j}$ of $G$.

2. Minimize $\phi:=\|M\|_{F}^{2}$ with respect to all effective parameter elements $g_{i j}$ of $G$ using standard numerical routines involving $\nabla_{G} \phi$ (compute with (21) and (22)) and/or $\nabla_{G}^{2} \phi$ (compute with (24) and (25)).

3. Choose $\hat{K}=\hat{G} T(\hat{G})^{-1}$ where $\hat{G}$ is the minimizer obtained in (2) as the robust deadbeat regulator gain.

\section{Numerical Example}

Consider the reachable pair $(A, B)$ where

$$
A=\left[\begin{array}{ccccc}
1 & 1 & 0 & 1 & 0 \\
0 & 0 & 1 & 0 & 0 \\
0 & -1 & 0 & 0 & 0 \\
0 & 0 & 0 & 1 & 0 \\
0 & 1 & 0 & 0 & 1
\end{array}\right] \quad, \quad B=\left[\begin{array}{lll}
0 & 1 & 0 \\
0 & 0 & 0 \\
1 & 0 & 0 \\
0 & 0 & 1 \\
0 & 0 & 1
\end{array}\right]
$$

The system matrices were also used by Klein (1984) and Sebakhy and Abdel-Moneim (1970) when considering deadbeat regulators. The controllability indices are 3,1 , and 1 . To have minimum regulation time with any initial state, the size of the Jordan blocks in $J$ should be chosen equal to the controllability indices. That is,

$$
J=\left[\begin{array}{lllll}
0 & 1 & 0 & 0 & 0 \\
0 & 0 & 1 & 0 & 0 \\
0 & 0 & 0 & 0 & 0 \\
0 & 0 & 0 & 0 & 0 \\
0 & 0 & 0 & 0 & 0
\end{array}\right]
$$

The initial guess $G(0)$ is taken as

$$
\left[\begin{array}{rrrrr}
-0.3600 & -0.1356 & -1.3493 & -1.2704 & 0.9846 \\
-0.0449 & -0.7989 & -0.7652 & 0.8617 & -0.0562 \\
0.5135 & 0.3967 & 0.7562 & 0.4005 & -1.3414
\end{array}\right]
$$

which is randomly generated from a normal distribution with unit variance and $T(G(0))$ is nonsingular. According to the discussion in Section 3.2, we have the elements in the last three columns of $G$ ineffective. That is, there are only six effective parameters in $G$. The minimization was carried out with analytical gradient and Hessian, eventually $\|M\|_{F}$ converges to the value 2.5495 and the corresponding feedback gain matrix is given by

$$
K_{\text {robust }}=\left[\begin{array}{ccccc}
0 & 0 & -1 & 1 & -1 \\
-1 & -1 & 0 & -1 & 0 \\
0 & -\frac{1}{2} & 0 & -\frac{1}{2} & -\frac{1}{2}
\end{array}\right]
$$

It can be shown that such $K_{\text {robust }}$ is the unique global minimizer of $\|A+B K\|_{F}$ with minimum value equals $\sqrt{13 / 2} \approx 2.54950976$. For the present example, all deadbeat regulation feedback gains are parametrized with the minimum number of parameters by

$$
K_{a l l}=\left[\begin{array}{ccccc}
0 & 0 & -1 & 1 & -1 \\
-1 & \alpha_{1}-1 & \alpha_{1}+\alpha_{3} & -\alpha_{1}-1 & \alpha_{1} \\
0 & \alpha_{2} & \alpha_{2}+\alpha_{4} & -\alpha_{2}-1 & \alpha_{2}
\end{array}\right]
$$

for some real numbers $\alpha_{i}, i=1, \ldots, 4$. To illustrate the robustness of the solution, we compare the $K_{\text {robust }}$ with a deadbeat regulator gain,

$$
K_{\text {random }}=\left[\begin{array}{ccccc}
0 & 0 & -1 & 1 & -1 \\
-1 & -2.84 & -3.17 & 0.84 & -1.84 \\
0 & -3.72 & -2.87 & 2.72 & -3.72
\end{array}\right]
$$

obtained by choosing $\alpha_{1}=-1.84, \alpha_{2}=-3.72, \alpha_{3}=$ -1.33 , and $\alpha_{4}=0.85$, drawn from a normal distribution of zero mean and unit variance. In this case, $\left\|A+B K_{\text {random }}\right\|_{F}=10.2260$.

Consider the perturbed closed-loop state matrix

$$
A+B K+\mu \Delta
$$

where $\Delta$ is a random perturbation (elements are independent and Gaussian distributed) normalized such that $\|\Delta\|_{F}=1$, and $\mu>0$ is a parameter controlling the level of perturbation. The associated measure of the degree of perturbation on the spectrum is given by

$$
d_{\mu}(\Delta):=\max _{i}\left|\lambda_{i}(A+B K+\mu \Delta)\right|
$$

where $\lambda_{i}(\cdot)$ denotes the $i$ th eigenvalue of $(\cdot)$. This measure of perturbation is equivalent to the optimal matching distance (Stewart and Sun 1990). When $d_{\mu}(\Delta)>1$, the perturbation leads to instability in the closed-loop system. The results of 1000 random $\Delta s$ are summarized in Table 1. In Table 1, the worst case, mean, and the standard deviation of $d_{\mu}$ over the 1000 trials (common to $K_{\text {robust }}$ and $K_{\text {random }}$, and $\mu=0.01,0.1$, and 0.2 ) are recorded. It can be observed that the robust deadbeat regulator gain $K_{\text {robust }}$ always performs better then $K_{\text {random }}$ in the three chosen levels of perturbation. At $\mu=0.2, K_{\text {random }}$ gives unstable closed-loop in some cases. By considering the 1000 samples in each case, it can be concluded that the mean of $d_{\mu}$ for $K_{\text {robust }}$ is significantly better than that of $K_{\text {random }}$ at the level of $0.1 \%$ in a statistical sense.

\section{Conclusion}

In this work, we have formulated the robust deadbeat regulation problem as an unconstrained optimization problem. A new objective function is introduced which, in contrast to the measures of robustness defined for this type of pole assignment problem, does not require the computation of eigenvectors. Moreover, the objective function has analytical gradient and Hessian which can be evaluated efficiently through matrix manipulations. The numerical example demonstrated the good performance of the robust deadbeat regulator gain in face of perturbation in the closed-loop matrix. 


\begin{tabular}{||c|cc|cc|cc||}
\hline$d_{\mu}$ & \multicolumn{2}{|c|}{$\mu=0.01$} & \multicolumn{2}{c|}{$\mu=0.1$} & \multicolumn{2}{c||}{$\mu=0.2$} \\
\hline & $K_{\text {robust }}$ & $K_{\text {random }}$ & $K_{\text {robust }}$ & $K_{\text {random }}$ & $K_{\text {robust }}$ & $K_{\text {random }}$ \\
worst & 0.2518 & 0.3196 & 0.5561 & 0.8123 & 0.7204 & 1.1330 \\
mean & 0.1572 & 0.2007 & 0.3628 & 0.5058 & 0.4734 & 0.6817 \\
stand. dev. & 0.0403 & 0.0437 & 0.0813 & 0.1098 & 0.1009 & 0.1546 \\
\hline
\end{tabular}

Table 1. Comparison of Results

\section{References}

Bhattacharyya, S. P. and E. de Souza (1982). Pole assignment via Sylvester equation. Systems and Control Letters 1(4), 261-263.

Byers, R. and S. G. Nash (1989). Approaches to robust pole assignment. Int. J. Control 49, 97-117.

Cavin III, R. K. and S. P. Bhattacharyya (1983). Robust and well-conditioned eigenstructure assignment via Sylvester's equation. Optim. Control Appl. and Meths. 4, 205-212.

Chatelin, F. (1993). Eigenvalues of matrices. Chichester: Wiley.

Emami-Naeini, A. and G. Franklin (1982). Deadbeat control and tracking of discrete time systems. IEEE Trans. Automatic Control AC-27(2), 176-180.

Fahmy, M. M. and J. O'Reilly (1983a). Dead-beat control of linear discrete-time systems. Int. J. Control 37(4), 685-705.

Fahmy, M. M. and J. O'Reilly (1983b). Use of the design freedom of time-optimal control. Systems and Control Letters 3, 23-30.

Farison, J. B. and F. C. Fu (1970). The matrix properties of minimum time discrete linear regulator control. IEEE Trans. Automat. Contr. AC-15, 390391.

Goodwin, G. C. and K. S. Sin (1984). Adaptive Filtering Prediction and Control. New Jersey: PrenticeHall.

Gourishankar, V. and K. Ramar (1976). Pole assignment with minimum eigenvalue sensitivity to plant parameter variations. Int. J. Control 23, 493-504.

Kaczorek, T. (1992). Linear Control Systems: Vol.1 Analysis of Multivariable Systems. England: Research Studies Press.

Kalman, R. E. and J. E. Bertram (1958). General synthesis procedure for computer control of single-loop and multi-loop linear systems. AIEE Trans. Applications and Industry $77,602-609$.

Kautsky, J. and N. K. Nichols (1985). Robust multiple eigenvalue assignment by state feedback in linear systems. Contemporary Mathematics 47, 253-264.

Kautsky, J., N. K. Nichols, and P. van Dooren (1985). Robust pole assignment in linear state feedback. Int. J. Control 41, 1129-1155.

Klein, G. (1984). On the relationship between controllability indexes, eigenvector assignment, and deadbeat control. IEEE Trans. Automat. Contr. AC29(1), 77-79.
Kučera, V. (1971). The structure properties of timeoptimal discrete linear control. IEEE Trans. Automat. Contr. AC-16, 375-377.

Kučera, V. and M. Šebek (1984). On deadbeat controllers. IEEE Trans. Automat. Contr. AC-29(8), 719-722.

Lam, J. and W. Yan (1995). A gradient flow approach to robust pole-placement problem. Int. J. of Robust and Nonlinear Control 5(3), 175-185.

Leden, B. (1977). Multivariable dead-beat control. Automatica 13, 185-188.

Mullis, C. T. (1972). Time optimal discrete regulator gains. IEEE Trans. Automatic Control AC-17, 265266.

Owens, T. J. and J. O'Reilly (1989). Parametric state-feedback control for arbitrary eigenvalue assignment with minimum sensitivity. IEE Proc. D 136(6), 307-313.

Petkov, P. H., N. D. Christov, and M. M. Konstantinov (1991). Computational Methods for Linear Control Systems. London: Prentice-Hall.

Schlegel, M. (1982). Parameterization of the class of deadbeat controllers. IEEE Trans. Automatic Control $A C-27(3), 727-729$.

Sebakhy, O. A. and T. M. Abdel-Moneim (1970). State regulation in linear discrete-time systems in minimum time. IEEE. Trans. Automat. Contr. $A C$ $24(2), 84-87$.

Stewart, G. W. and J. Sun (1990). Matrix Perturbation Theory. Academic Press.

van Dooren, P. (1984). Deadbeat control: a special inverse eigenvalue problem. BIT 24, 517-719.

White, B. A. (1995). Eigenstructure assignment: a survey. Proc. I.Mech.E, Part I 209(I1), 1-11. 